\title{
Evaluation of Association between
} Chlamydophila pneumoniae and Atherosclerotic Plaques in Coronary Artery Disease Patients with Abnormal Angiography

\author{
Darioush Iranpour1, Frahad Abbasi2 ${ }^{*}$, Kamran Mirzayee1, Nahid Mohammadzadeh', \\ Alireza Raeisi' ${ }^{1}$, Akram Najafi ${ }^{3}$ \\ ${ }^{1}$ Bushehr University of Medical Science, Bushehr, Iran \\ ${ }^{2}$ The Persian Gulf Tropical Medicine Research Center, Bushehr University of Medical Sciences, Bushehr, Iran \\ ${ }^{3}$ The Persian Gulf Marine Biotechnology Research Center, Bushehr University of Medical Sciences, Bushehr, \\ Iran \\ Email: ${ }^{*}$ abbasi55@yahoo.com
}

Received 11 August 2014; revised 26 September 2014; accepted 11 October 2014

Copyright (C) 2014 by authors and Scientific Research Publishing Inc.

This work is licensed under the Creative Commons Attribution International License (CC BY).

http://creativecommons.org/licenses/by/4.0/

(c) (i) Open Access

\begin{abstract}
The role of Chlamydophila pneumoniae in pathogenesis of atherosclerosis is one of the most important discussions in coronary artery diseases. In this study, the relationship between $C$. pneumoniae seropositivity and atherosclerotic plaque was evaluated among two groups: one group with significant coronary stenosis and one group with normal coronary angiography. Serum $C$. pneumonia IgM and IgG were evaluated and compared in case and control groups. The seropositivity rates of IgM and IgG antibodies were not statistically differ between case and control groups, although the incidences of positivity were more in case group. The results of our study did not show a correlation between $C$. pneumonia infection and atherosclerotic plaque or coronary artery stenosis neither in acute nor in chronic infection. More precise studies are needed to clarify the probable inflammatory cascade that starts with $C$. pneumonia infection and lead to development of atherosclerotic plaque.
\end{abstract}

\section{Keywords}

Chlamydophila pneumonias, Atherosclerosis, Angiography, Infection

\footnotetext{
*Corresponding author.
} 


\section{Introduction}

Atherosclerosis is induced by multiple mechanisms which consist of lipid metabolism imbalance, immune response disequilibrium, and chronic inflammation of the artery wall [1]. Several studies have shown a relationship between the development of atherosclerosis and the presence of infectious diseases [1] [2]. Chlamydophila pneumoniae and several other infectious agents have been reported with an increased risk of vascular damage. Helicobacter pylori, Porphyromonas gingivalis, influenza A virus, cytomegalovirus, herpes virus, hepatitis C virus, and human immunodeficiency virus are causative agents [3].

Infections may cause atherosclerosis with direct or indirect effects. Recently, studies have shown that these chronic infections are responsible for atherosclerosis, rather than the effects of a single organism. However, the role of infection, as a proinflammatory cause of atherosclerosis, is still controversial in the literature [1]. $C$. pneumoniae may play an important role in development of atherosclerosis and atherosclerotic plaque [2]. Generally C. pneumonia is one of important causes of pneumonia in human. It also can cause meningoencephalitis, arthritis and myocarditis.

The involvement of $C$. pneumoniae in chronic diseases may be due to its ability to induce persistent forms in which Chlamydophila remains viable but is not cultivable [4]. The mechanisms of atherosclerosis related to $C$. pneumoniae infection are not clear [5].

This study was aimed to evaluate the association between C. pneumoniae and atherosclerotic plaques in coronary artery disease patients with abnormal angiography in Iran.

\section{Materials and Methods}

In our study, the relationship between C. pneumoniae seropositivity and atherosclerotic plaque was evaluated. Other risk factors for atherosclerosis were also assessed in case and control groups. Case group were selected from patients with documented coronary artery disease, i.e. those who had atherosclerotic plaque and coronary stenosis more than $50 \%$ in at least two vessels. Control group were selected from patients with normal coronary angiograghy.

A total of 90 atherosclerotic patients and 90 control subjects without coronary diseases were included in this study. In our case group 71 patients (79\%) were male and 19 patients (21\%) were female. The average of age was 45 - 87 years with mean age of $65.3 \pm 8.7$ years. In our control group 41 patients (46\%) were male and 49 patients (54\%) were female. Although the difference of gender composition in case and control groups may affect the results, it seems that role of other factors were stronger. The average of age was 42 - 84 years with mean age of $61.6 \pm 9.6$ years. Both groups were also evaluated for the presence of risk factors such as age, gender, smoking, hypertension, diabetes, dyslipidemia, family history of coronary artery diseases and the high levels of triglyceride and cholesterol (total, HDL and LDL), ESR, CRP, leukocytosis and thrombocytosis (as acute phase reactants).

Triglyceride and cholesterol was evaluated by enzymatic and CRP was evaluated by immunoassay methods.

The presence of serum C. pneumoniae IgG and IgM antibodies were performed by ELISA methods. Although PCR method for detection of $C$. pneumonia DNA is more precise than serum antibody detection, this method was not performed because of difficulty of preparing specific kits. Echocardiography was preformed for all the patients of case and control groups. Data was analyzed by SSPS software using chi square and Mann Whitney tests. All steps of this study were approved by the Ethical Committee of Bushehr University of Medical Science.

\section{Results}

C. pneumoniae antibody seropositivity rate was found $10 \%$ (9/90) in case group by ELISA methods, while this rate in control group was about 7\% (6/90) (Table 1). Despite more patients with seropositivity in case group, statistically no significant difference between case and control groups was detected. $P$-value was 0.239 for IgM anticlamydophila antibody between case and control groups and was 0.121 for IgM anticlamydophila antibody between case and control groups.

Statistical analysis showed no significant differences between ESR, CRP and hemoglobin level of two groups. Suffering from Diabetes mellitus and hyperlipidemia (including level of triglyceride, LDL cholesterol and HDL cholesterol) were the same in case and control groups.

For all the patients echocardiography was performed. Statistical analysis showed no significant differences in 
Table 1. Level of anticlamydophila antibodies in case and control groups.

\begin{tabular}{cccccccc}
\hline Prevalence & HLP (percent) & $\begin{array}{c}\text { Smoker } \\
\text { (percent) }\end{array}$ & DM (percent) & $\begin{array}{c}\text { Abnl CRP } \\
\text { (percent) }\end{array}$ & $\begin{array}{c}\text { Mean WBC } \\
\text { level }\end{array}$ & $\begin{array}{c}\text { Mean TG level } \\
\text { (mg/dl) }\end{array}$ & $\begin{array}{c}\text { Mean Chol level } \\
\text { (mg/dl) }\end{array}$ \\
\hline Nl angiography pt. & 37.4 & 29.7 & 28.6 & 11 & 7062 & 159 & 182 \\
Abnl angiography pt. & 44.9 & 31.5 & 30.3 & 15.6 & 9299 & 190 & 188 \\
\hline
\end{tabular}

HLP: hyperlipidemia; DM: dianetes mellitus; CRP: C-reative protein; WBC: white blood cell; TG: triglyceride; Chol: cholesterol; pt: patient.

ejection fraction. There were significant differences in WBC and platelet levels of case and control groups. We also detected that patient group suffer from hypertension more than control group. The evaluation of the risk factors revealed that age, dyslipidemia, smoking, diabetes mellitus, ESR level, CRP levels, HDL and LDL cholesterol level and triglyceride level exhibited statistically no significant differences between patient and control groups (Table 2).

\section{Discussion}

Coronary artery disease is the leading cause of death in many countries. The underlying mechanism of the chronic inflammatory process in atherosclerosis and atherosclerotic plaque is still unclear. Many viruses and bacteria may be associated with atherosclerotic events and may have a trigger role [3]. Atherosclerosis is thought to be induced by chronic inflammatory process [6]. The synergism of infection with conventional cardiovascular risk factors in atherosclerosis is now debated. It is hypothesized that coronary arterial events may correlate with chronic infection and pathogen agents and is further aggravated by hypercholesterolemia [7]. It is not currently clear what phenomenon causes the chronic inflammation within atherosclerotic plaques.

One study suggests that infection with bacteria or viruses can contribute to the pathogenesis of atherosclerosis in direct way of vascular cells or in indirect way by affect of cytokines or acute phase proteins induced by infection at non-vascular sites. This paradigm has been supported by multiple epidemiological studies that have shown positive associations between the risk of cardiovascular disease morbidity and mortality and incidence of infection [8].

It has also been supported by experimental studies showing an acceleration of the development of atherosclerosis following infection of hyperlipidaemic animal models. There are now a large number of different infectious agents that have been linked with an increased risk of cardiovascular disease. C. pneumoniae, Porphyromonas gingivalis, Helicobacter pylori, influenza A virus, hepatitis $C$ virus, cytomegalovirus, and human immunodeficiency virus are microorganisms that may have role in developing atherosclerosis [3]. However, there are significant differences in the validity of the data supporting their association with cardiovascular disease pathogenesis. In some cases, the infectious agents are found within the plaques and viable organisms can be isolated suggesting a direct effect. In other cases, the association is entirely based on biomarkers [8].

The role of various microorganisms including $C$. pneumoniae, have been frequently investigated in the pathogenesis of atherosclerosis [9]. C. pneumoniae is an obligate intracellular respiratory pathogen for humans. Infection by C. pneumoniae may be linked etiologically to extra-respiratory diseases especially atherosclerosis [10].

The atherogenic actions of $C$. pneumoniae are dependent upon a high-cholesterol environment in vivo. It is possible that oxidized low-density lipoprotein (oxLDL) is responsible for promoting the atherogenic effects of $C$. pneumoniae through a stimulation of cell proliferation [11]. Recently, it has been demonstrated that C. pneumoniae up-regulates expression of the lectin-like ox-LDL receptor (LOX-1) in endothelial cells. Many of the proatherogenic effects of ox-LDL occur through its activation and uptake by LOX-1 [12].

Al-Bannawi's study demonstrated an interactive link between C. pneumoniae and platelets in atherosclerotic patients, leading to induction of potential chemokines and possibly disease development [13]. In order for $C$. pneumoniae to play a causative role in chronic human disease, it would need to persist within infected tissue for extended periods of time. Current theory suggests that $C$. pneumoniae may persist at the site of infection via an alternative replicative form, known as an aberrant body. The protein antigens of persistent C. pneumoniae infection found in the atheromatous lesions from patients in this study could potentially be used as markers to detect such infections and some may be virulence factors or immunogens specific to C. pneumoniae, thus serving as target molecules for diagnostic use or therapeutic intervention [14]. 
Table 2. Prevalence of different parameters in case and control groups.

\begin{tabular}{ccc}
\hline \multicolumn{1}{c}{$\begin{array}{c}\text { Anticalymophila } \\
\text { antibodies }\end{array}$} & Mean IgM level & Mean IgG level \\
Patients & 3.99 & 2.96 \\
\hline Nl angiography pt. & 3.44 & 3.46 \\
\hline Abnl angiography pt. & \\
\hline
\end{tabular}

Halvorsen's study on 57 patients with carotid artery plaques indicated that persistent $C$. pneumoniae is not a common phenomenon in ones with carotid atherosclerosis [15]. Since the experimental and clinical research of influence of inflammations on the development of atherosclerosis progressed a lot but no exact evidence of this complicated pathogenic mechanism was found. It will obviously take some time to confirm whether the relation between infections and artherosclerosis is causal, i.e. initiating the pathogenic process, accelerating it or keeping it alive [6].

In Dogra's study the hypothesis that infection plays a role in atherosclerosis was evaluated; patients in case group received $500 \mathrm{mg}$ of oral azithromycin once daily for 5 days, while the control group did not have azithromycin and there was a positive correlation between azithromycin and secondary prevention of coronary artery disease [16].

In this study we choose our case group from patients with documented coronary artery disease, i.e. those who had atherosclerotic plaque and coronary stenosis more than $50 \%$ in at least two vessels. By this choosing the involvement of coronary artery was obvious and other factor can be evaluated according to it. C. pneumoniae was assessed by using IgM seroevaluation that shows acute infection and IgG seroevaluation that indicates chronic infection or infection in the past. The results of our study could not make a correlation between C. pneumoniae infection and atherosclerotic plaque or coronary artery stenosis neither in acute nor in chronic infection.

\section{Conclusion}

Despite this result we cannot deny the probable role of this organism in developing atherosclerosis that may be due to an indirect effect not a direct one. Precise studies are needed to clarify that the probable inflammatory cascade starts with C. pneumoniae infection and lead to development of atherosclerotic plaque.

\section{Acknowledgements}

All authors are grateful to the Bushehr University of Medical Science, for their executive support of this project.

\section{References}

[1] Tufano, A., Di Capua, M., Coppola, A., Conca, P., Cimino, E., Cerbone, A.M. and Di Minno, G. (2012) The Infectious Burden in Atherothrombosis. Seminars in Thrombosis and Hemostasis, 38, 515-523. http://dx.doi.org/10.1055/s-0032-1315759

[2] Luque, A., Turu, M.M., Rovira, N., Juan-Babot, J.O., Slevin, M. and Krupinski, J. (2012) Early Atherosclerotic Plaques Show Evidence of Infection by Chlamydia pneumoniae. Frontiers in Bioscience (Elite Edition), 4, $2423-2432$. http://dx.doi.org/10.2741/E554

[3] Al-Ghamdi, A., Jiman-Fatani, A.A. and El-Banna, H. (2011) Role of Chlamydia pneumoniae, Helicobacter pylori and Cytomegalovirus in Coronary Artery Disease. Pakistan Journal of Pharmaceutical Sciences, 24, 95-101.

[4] Di Pietro, M., Tramonti, A., De Santis, F., De Biase, D., Schiavoni, G., Filardo, S., Zagaglia, C. and Sessa, R. (2012) Analysis of Gene Expression in Penicillin G Induced Persistence of Chlamydia pneumoniae. Journal of Biological Regulators \& Homeostatic Agents, 26, 277-284.

[5] Zhang, L., Li, X., Zhang, L., Wang, B., Zhang, T. and Ye, J. (2012) Chlamydophila (Chlamydia) Pneumoniae Infection Promotes Vascular Smooth Muscle Cell Adhesion and Migration through IQ Domain GTPase-Activating Protein 1. Microbial Pathogenesis, 53, 207-213. http://dx.doi.org/10.1016/j.micpath.2012.07.005

[6] Zeman, K. (2006) Atherosclerosis and Infection. Vnitrni Lekarstvi, 52, 823-826.

[7] Birck, M.M., Pesonen, E., Odermarsky, M., Hansen, A.K., Persson, K., Frikke-Schmidt, H., Heegaard, P.M. and Liuba, P. (2011) Infection-Induced Coronary Dysfunction and Systemic Inflammation in Piglets Are Dampened in Hypercholesterolemic Milieu. American Journal of Physiology-Heart and Circulatory Physiology, 300, 1595-1601. 
http://dx.doi.org/10.1152/ajpheart.01253.2010

[8] Rosenfeld, M.E. and Campbell, L.A. (2011) Pathogens and Atherosclerosis: Update on the Potential Contribution of Multiple Infectious Organisms to the Pathogenesis of Atherosclerosis. Thrombosis and Haemostasis, 106, 858-867. http://dx.doi.org/10.1160/TH11-06-0392

[9] Kılıç, Z.B., Poyraz, O. and Kılıç, A.T. (2012) Investigation of Chlamydophila pneumoniae Seropositivity and Risk Factors in Patients with Atherosclerotic Vascular Disease in Sivas, Turkey. Mikrobiyoloji Bulteni, 46, 156-158.

[10] Eddens, T., Beaudoin, S., Steinberger, A., Little, C.S., Shell, D., Wizel, B., Balin, B. and Fresa-Dillon, K.L. (2012) Effect of Age and Vaccination on Extent and Spread of Chlamydia pneumoniae Infection in C57BL/6 Mice. Immunity \& Ageing, 9, 11. http://dx.doi.org/10.1186/1742-4933-9-11

[11] Chahine, M.N., Deniset, J., Dibrov, E., Hirono, S., Blackwood, D.P., Austria, J.A. and Pierce, G.N. (2011) Oxidized LDL Promotes the Mitogenic Actions of Chlamydia pneumoniae in Vascular Smooth Muscle Cells. Cardiovascular Research, 92, 476-483. http://dx.doi.org/10.1093/cvr/cvr251

[12] Campbell, L.A., Puolakkainen, M., Lee, A., Rosenfeld, M.E., Garrigues, H.J. and Kuo, C.C. (2012) Chlamydia pneumoniae Binds to the Lectin-Like Oxidized LDL Receptor for Infection of Endothelial Cells. Microbes and Infection, 14, 43-49. http://dx.doi.org/10.1016/j.micinf.2011.08.003

[13] Al-Bannawi, A., Al-Wesebai, K., Taha, S. and Bakhiet, M. (2011) Chlamydia pneumoniae Induces Chemokine Expression by Platelets in Patients with Atherosclerosis. Medical Principles and Practice, 20, 438-443. http://dx.doi.org/10.1159/000324553

[14] Borel, N., Pospischil, A., Dowling, R.D., Dumrese, C., Gaydos, C.A., Bunk, S., Hermann, C., Ramirez, J.A. and Summersgill, J.T. (2012) Antigens of Persistent Chlamydia pneumoniae within Coronary Atheroma from Patients Undergoing Heart Transplantation. Journal of Clinical Pathology, 65, 171-177. http://dx.doi.org/10.1136/jclinpath-2011-200270

[15] Halvorsen, D.S., Karlsen, J., Notø, A.T., Mathiesen, E.B., Njølstad, I., Gutteberg, T.J., Vorland, L.H. and Hansen, J.B. (2007) No Detectable Chlamydia pneumoniae and Cytomegalovirus DNA in Leukocytes in Subjects with Echolucent and Echogenic Carotid Artery Plaques. International Journal of Cardiology, 117, 388-394. http://dx.doi.org/10.1016/j.ijcard.2006.05.025

[16] Dogra, J. (2012) Oral Azithromycin in Extended Dosage Schedule for Chronic, Subclinical Chlamydia pneumoniae Infection Causing Coronary Artery Disease: A Probable Cure in Sight? Results of a Controlled Preliminary Trial. International Journal of General Medicine, 5, 505-509. http://dx.doi.org/10.2147/IJGM.S31625 
Scientific Research Publishing (SCIRP) is one of the largest Open Access journal publishers. It is currently publishing more than 200 open access, online, peer-reviewed journals covering a wide range of academic disciplines. SCIRP serves the worldwide academic communities and contributes to the progress and application of science with its publication.

Other selected journals from SCIRP are listed as below. Submit your manuscript to us via either submit@scirp.org or Online Submission Portal.
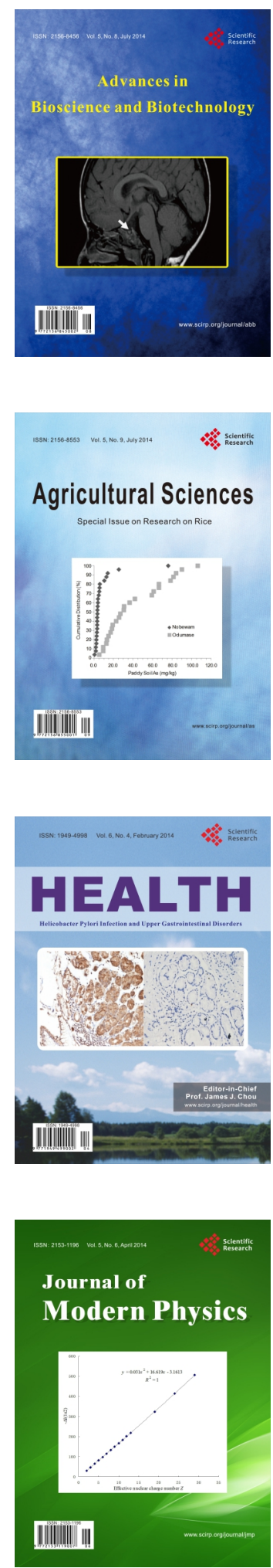
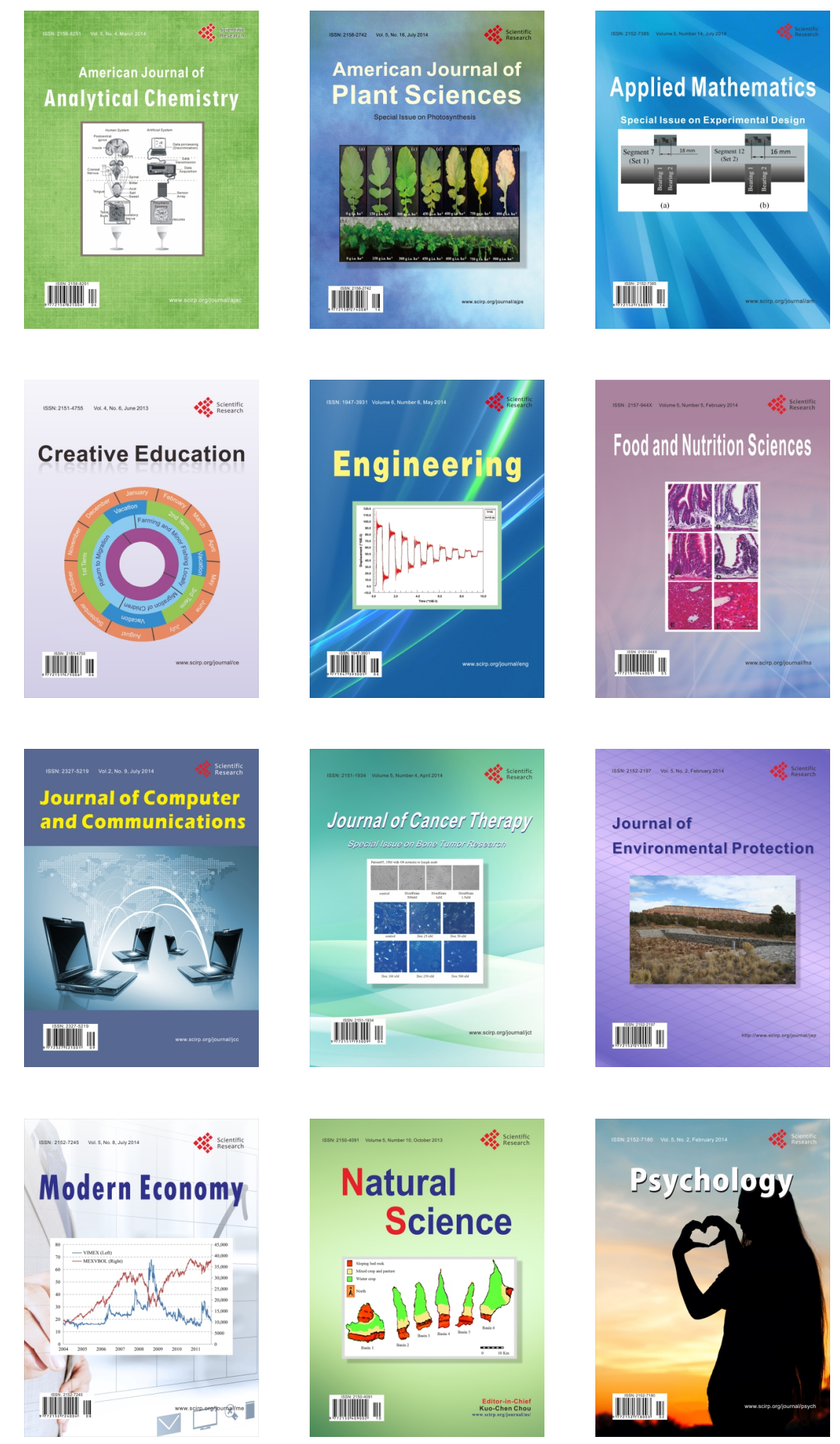